\title{
PARTITIONS OF THE NATURAL NUMBERS
}

\author{
Myer Angel ${ }^{1)}$ \\ (received September 23, 1963)
}

1. We obtain in this article some results concerning partitions of the natural numbers, the most important of which is a generalization of that quoted immediately below. Some intuitive material is included.

In 1954, J. Lambek and L. Moser [1] showed that "Two non-decreasing sequences $f$ and $g$ (of non-negative integers) are inverses if and only if the corresponding sets $F$ and $G$ of positive integers, defined by $F(m)=$ the $m$ th element of $F=f(m)+m$ and $G(n)=g(n)+n$ are complementary. "The inverse of $f$ was defined so that it coincided with $f^{+}$, where $\mathrm{f}^{+}(\mathrm{n})=$ the number of $\mathrm{m}$ such that $\mathrm{f}(\mathrm{m})<\mathrm{n}$. Thus the complementary sets $F=\{2,6,12,20,30, \ldots\}$ and $G=\{1,3,4$, $5,7, \ldots\}$ defined by $F(m)=m^{2}+m$ and $G(n)=[\sqrt{n-1}]+n$ correspond to the inverse sequences $m^{2}$ and $[\sqrt{n-1}]$.

Using this example, a possible geometric "map" of the concepts involved in the theorem is given in Figs. 1 and 2 . Thus, in Fig. 1, $\{n+f(n) \mid n=1,2,3, \ldots\}$ is represented by two vertical (number) lines, each containing an infinity of (natural) points, spaced at equal distances, the analogue of + being a rising bar which straddles the lines. Understanding the theorem may be largely equivalent to tracing out this, or the reader's own, diagram mentally, until any part suggests the whole.

1) Editor's comment. Mr. Angel is an undergraduate student at McGill University. This paper was written when he was a second year student.

Canad. Math. Bull. vol. 7, no. 2, April 1964 


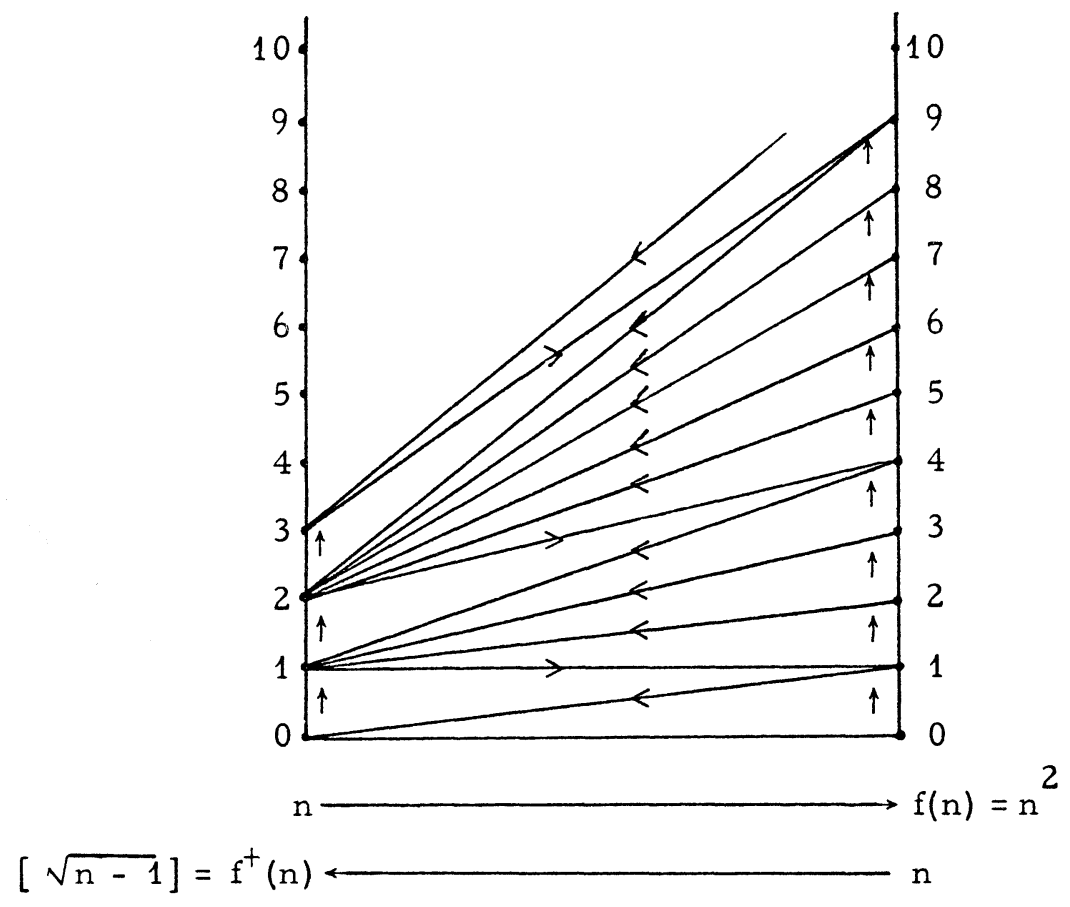

Fig. 1

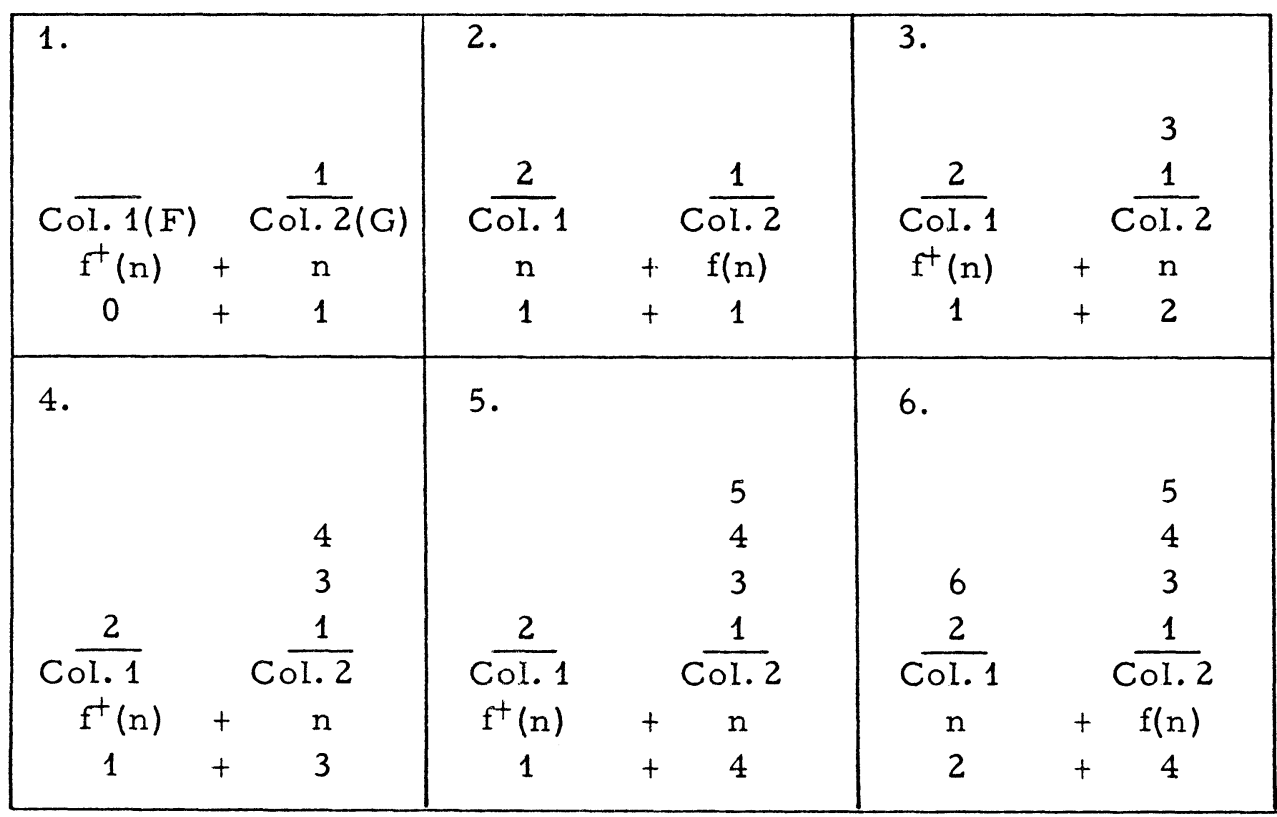

Fig. 2 
In Fig. 2, the mth natural number is entered in an $n$th position in Column 1 (representing the set $F$ ), if there have been $f(n)$ entries in Column 2 (representing the set $G$ ). It is then of the form $n+f(n)$. It is entered in an nth position in Column 2 if there have been $f^{+}(n)$ entries in Column 1. It is then of the form $f^{+}(n)+n$. One and only one of the se conditions holds for each $\mathrm{m}$.

$\begin{array}{rrrrrrrrrrrr} & 1 & & & & 1 & & & & & \\ 2 & 1 & 2 & 2 & 2 & 1 & 2 & 2 & 2 & 2 & 2 & 1 \\ \uparrow & \uparrow & \uparrow & \uparrow & \uparrow & \uparrow & \uparrow & \uparrow & \uparrow & \uparrow & \uparrow & \uparrow \\ 1 & 2 & 3 & 4 & 5 & 6 & 7 & 8 & 9 & 10 & 11 & 12\end{array}$

Fig. 3

In Fig. 3, a sequence of $1^{\prime} \mathrm{s}$ is formed. $2^{\prime} \mathrm{s}$ are interpolated so that before the nth 1 , there are $f(n) 2^{\prime} s$. If the $m$ th term in the sequence is an nth 1 , let $m$ be in $F$. Then $F(n)=m=$ number of $1^{\prime} s+$ number of $2^{\prime} s=n+f(n)$. If the $m$ th term of the sequence is an $n$th 2 , let $m$ be in $G$. Then $G(n)=m=$ number of $2^{\prime} s+$ number of $1^{\prime} s=n+f^{+}(n)$, as is easily verified.

The assignment of natural numbers to complementary sets, given $f$, may be considered as being made either inductively as in Fig. 2, or after an interpolation as in Fig. 3; this latter method proves to be the more useful in the general case we shall be discussing. Sequences of numerals representing sets (such as in Fig. 3) a re linguistically more amenable to treatment by interpolation than are sets of numbers (such as in Fig. 2), and will hence be introduced when needed.

We observe that the "complementary" sets are " 2 " sets, and that dichotomy is a special case of partition. This is amplified in Fig. 4, although such particular imagery as is there presented should serve only as the stepping-stone to an understanding which dispenses with it. The intuitive approach will therefore be exchanged afterwards for a set-theoretical formulation in which the temporal and kinetic elements are camouflaged in static, symbolic representation. 


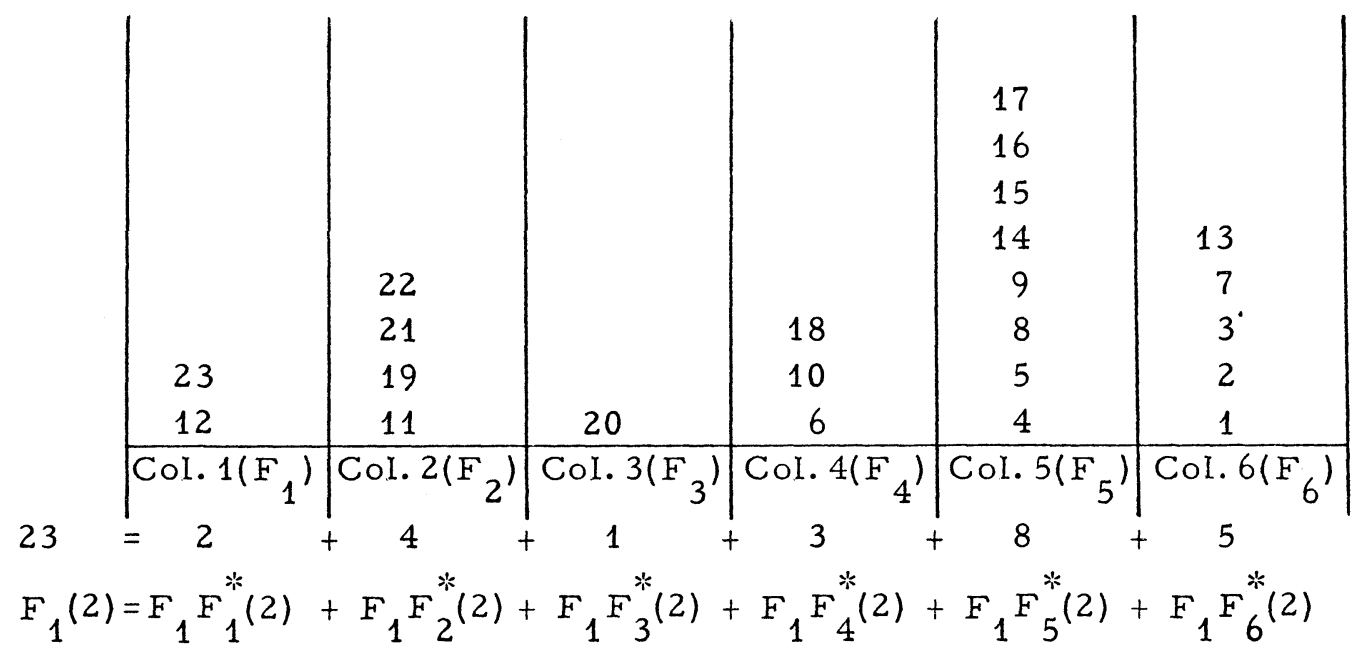

Fig. 4

Fig. 4 is interpreted as follows. As the natural numbers are counted off, they are entered in various columns which grow. The natural numbers may be classified according to the columns to which they are assigned. Each entry may be expressed as the number that have so far been entered in its column, plus the corresponding (functionally determined) number of previous entries in the other columns (cf. $\S 5$, Ex. 1, and Fig. 6).

2. In the following, $\mathrm{N}$ denotes the natural numbers $1,2,3, \ldots$ and $S_{n}$ the first $n$ natural numbers $1,2,3$, $\ldots, n$. Partitions of $\mathrm{N}$ into a finite number of subsets are denoted by $\left\{F_{1}, F_{2}, F_{3}, \ldots, F_{n}\right\}$ or $\left\{F_{i} \mid i \in S_{n}\right\}$. The union, $F_{1} \cup F_{2} \cup F_{3} \ldots \cup F_{n}$, of the sets $F_{i}$ is $N$, and the intersection $F_{i} \cap F_{j}$ of $F_{i}$ and $F_{j}(i \neq j)$ is empty. The nth smallest element of a set is denoted by $F(n)$. Since the sets $F_{i}$ are also the ranges of strictly monotonic increasing functions on $\mathrm{N}$, they are often treated as such functions, which form a subclass of all sequences. Strictly monotonic increasing sequences are denoted by $F, G, \ldots$, and more generally nondecreasing sequences by $f, g, \ldots$ Some non-decreasing sequences which will appear below are $p_{n}$, the nth prime; $\pi(n)$, the number of primes not exceeding $n$; and $[r(n)]$, the 
greatest integer in $r(n)$ where $r$ is a non-decreasing realvalued function on $N$. We will say $f=g, f<g$, and $f \leq g$ if and only if $f(n)=g(n), f(n)<g(n)$, and $f(n) \leq g(n)$, respectively, for all $n \in N$.

The composition product of functions, which is the only product we shall use, is written $\mathrm{fgh} . . ., \mathrm{fgh}(\mathrm{n})$ being equivalent to $h(g(f(n)))$. (Those readers who prefer the usual composition notation, slightly inconvenient here, in which there is a psychological "set" provided by reading the functions in an order opposite to that in which they are calculated, will read the function products from right to left.) $f+g$ represents the sum of functions, not sets. I is the identity function, $S$ the successor $(n \rightarrow n+1)$ function and $P$ the predecessor $(n \rightarrow n-1)$ function.

$f^{*}$ is the function determined by $f^{*} f \leq I$ and $f^{*} S f>I$; i. e., $f^{* *}(n)$ is the number of members of $\{x \mid f(x) \leq n\}=$ $\{x \mid f(x)<n+1\}$, so that $S^{+}=f^{*}$ and $f^{+}=\mathrm{Pf}^{*}$.

We will adjoin 0 and $\infty$ to $N$ (more specifically, to the ranges of functions $f)$ for certain purposes. 0 is useful since it gives meaning to the $P$ function at 1 , and, in its capacity as additive identity, it signifies "nothing" or "no contribution to the sum". All negative integers are treated as 0 , and the value of any function at 0 is $0 . \infty$ is useful, since if any function becomes constant at $k$, i. e. beyond some point of its domain, then we may say $f^{+}(k)=\infty$. Rather than run a parallel rewording of the proofs below to cover the infinite case, we will allow the reader to check that this case has no essential distinction.

Some remarks on the behaviour of the functions we have defined, supplementing the standard algebra of functions, will facilitate the discussion below.

1) $\mathrm{FF}^{*}=\mathrm{I}$, since there are $\mathrm{n}$ elements of $\mathrm{F}$ which do not exceed $F(n)$, the nth element of $F$. On the other hand $F^{*} F=I$ only on $F$. There $F\left(F^{*} F\right)=\left(F F^{*}\right) F=I F=F$. Otherwise $F^{*} F<I$.

2) $\mathrm{FPF}^{*}=\mathrm{P}$, since $\mathrm{n}-1$ elements of $\mathrm{F}$ do not exceed $F(n)-1$. However, if $F$ and $G$ are complementary sets, 
$\mathrm{FPG}^{*}=\mathrm{FG}^{*}$ since all elements of $\mathrm{G} \leq \mathrm{F}(\mathrm{n})$ are $\leq F(n)-1$. $(f g)^{*}=g^{*} f^{*}$.

3) $\underset{g^{*}}{\mathrm{~g}} \mathrm{f}^{*} \mathrm{fg} \leq \mathrm{g}{ }^{*} \mathrm{~g} \leq \mathrm{I}$, and $\mathrm{g} \mathrm{f}^{*} \mathrm{Sfg} \geq \mathrm{g}{ }^{*} \mathrm{Sg}>\mathrm{I}$. Hence

4) It is shown in [1] that $\left(\mathrm{f}^{+}\right)^{+}=f$ which is the same as $\left(P(P f)^{*}\right)^{*}=f$. Then $\left(f^{*}\right)^{*}=P S\left(f^{*}\right)^{*}=P\left(f^{*} P\right)^{*}=P\left(P S f^{*} P\right)^{*}$ $=P\left(P(S f P)^{*}\right)^{*}=S f P$. This result may be obtained directly by observing that $(\mathrm{SfP}) \mathrm{f}^{*}=\mathrm{SP} \leq \mathrm{I}$ and $(\mathrm{SfP}) \mathrm{Sf}^{*}=\mathrm{Sff}^{*}=\mathrm{S}>\mathrm{I}$.

5) $\left(\mathrm{fg}^{*}\right)^{+}=\mathrm{P}\left(\mathrm{fg}^{*}\right)^{*}=\mathrm{Pg} \mathrm{f}^{* * *}=\mathrm{P}(\mathrm{SgP}) \mathrm{f}^{*}=\mathrm{gf}^{*}$.

6) $\mathrm{fg}^{*} \mathrm{~g} \leq \mathrm{f} \leq \mathrm{fg}{ }^{*} \mathrm{Sg}$; therefore $\mathrm{fg}^{*} \mathrm{gh}^{*} \leq \mathrm{f} \leq \mathrm{fg}^{*} \mathrm{Sgh}^{*}$.

3. If $\left\{F_{1}, F_{2}\right\}$ is a partition of $N$, then $F_{1}^{*}+F_{2}^{*}=I$, since the $n$ natural numbers not exceeding $n$ are all either in $F_{1}$ or in $F_{2}$. More generally, if $\left\{F_{i} \mid i \in S_{n}\right\}$ is a partition of $N$, then $\sum_{i \in S_{n}} F_{i}^{*}=I$. This equation represents an equality of functional values for all $n \in N$, and hence for any subset of $N$; thus $F=F \sum_{i \in S_{n}} F_{i}^{*}=\sum_{i \in S_{n}} F F_{i}^{*}$. (The observation that the nth natural number is equal to $\mathrm{n}$ is central to many of the remarks thus far. This equation of cardinal and ordinal values is characteristic of the natural numbers, so that a straightforward extension of the results below to other systems is unlikely, although analogous developments involving a substantial change in character might be attempted.)

If $\left\{F_{1}, F_{2}\right\}$ and $\left\{F_{3}, F_{4}\right\}$ are partitions of $N$, then $\left\{F_{1} F_{3}, F_{1} F_{4}, F_{2} F_{3}, F_{2} F_{4}\right\}$ is a partition of $N$, since every $n$ in $N$, the domain of $F_{3}$ and $F_{4}$, is either in $F_{1}$ or in $F_{2}$. More generally if $\left\{F_{i} \mid i \in S_{n}\right\},\left\{F_{j} \mid j \in S_{n}\right\},\left\{F_{k} \mid k \in S_{n}\right\}, \ldots$ are partitions of $N$, then so is $\left\{F_{i} F_{j} F_{k} \ldots \mid(i, j, k, \ldots)\right.$ $\left.\epsilon S_{n} \times S_{n} \times S_{n} \times \ldots\right\}$ where $S_{n} \times S_{n}$ is a Cartesian product, and some of the $F^{\prime} s$ may be empty. Each $F$ may be expressed as $\sum_{i \in S_{n}} F F_{i}^{*}$ so that we now have a wide class of partitions. 
Examples: Let $F_{1}$ be the set of squares, $F_{1}(n)=n^{2}$ $F_{2}$, the set of non-squares $F_{2}(n)=n+\{\sqrt{n}\} ; F_{3}$, the set of triangular numbers, $F_{3}(n)=n(n+1) / 2$; and $F_{4}$, the set of non-triangular numbers, $F_{4}(n)=n+\{\sqrt{2 n}\} \cdot(\{\}$ is the "closest integer" function.)

Two of the possible partitions obtainable from $\left\{F_{1}, F_{2}\right\}$ and $\left\{F_{3}, F_{4}\right\}$ are $\left\{F_{1} F_{3}, F_{1} F_{4}, F_{2} F_{3}, F_{2} F_{4}\right\}$ $=\left\{\left\{n^{2}\left(n^{2}+1\right) / 2\right\}, \quad\left\{n^{2}+\left\{\sqrt{\left(2 n^{2}\right)}\right\}\right\},\{(n+\{\sqrt{n}\})(n+\{\sqrt{n}\}+1) / 2\}\right.$, $\{n+\{\sqrt{n}\}+\{\sqrt{(2(n+\{\sqrt{n}\}))}\}\}$ and $\left\{F_{1} F_{1} F_{1}, F_{1} F_{1} F_{2}\right.$, $\left.F_{1} F_{2} F_{1}, F_{2} F_{1} F_{1}, F_{1} F_{2} F_{2}, F_{2} F_{1} F_{2}, F_{2} F_{2} F_{1}, F_{2} F_{2} F_{2}\right\}$ $=\left\{\left\{n^{8}\right\},\left\{n^{4}+n^{2}\right\},\left\{\left(n^{2}+n\right)^{2}\right\},\left\{(n+\{\sqrt{n}\})^{4}\right\}\right.$, $\left\{n^{2}+n+\left\{\sqrt{\left(n^{2}+n\right)}\right\}\right\}, \quad\left\{(n+\{\sqrt{n}\})^{2}+n+\{\sqrt{n}\}\right\}$, $\left\{\left(n+\{\sqrt{n}\}+\{\sqrt{(n+\{\sqrt{n})\}})^{2}\right\}\right.$, $\{n+\{\sqrt{n}\}+\{\sqrt{(n+\{\sqrt{n}\})}\}+\{n+\{\sqrt{n}\}+\{\sqrt{(n+\{\sqrt{n}}\})\}$

$$
+\{\sqrt{(n+}+\{\sqrt{n}\}+\{\sqrt{(n+\{\sqrt{n}\}})\})\}\} \text {. }
$$

It is understood that $\{f(n)\}=\{f(n) \mid n \in N\}$ when the braces clearly do not denote the "closest integer to" function.

The descriptions of $\mathrm{F}_{2}$ and $\mathrm{F}_{4}$ in terms of familiar functions were found in [1] by a method which may be generalized as follows: Let $\left\{F_{i} \mid i \in S_{n}\right\}$ be a partition of which $F_{1}$ is a subset. Then $F_{1}=\sum_{i=1}^{n} F_{1} F_{i}^{*}=\sum_{i=1}^{n}\left(F_{i=1} F^{*}\right)^{+}=F_{1} F_{1}^{*}$ $+\sum_{i=2}^{n}\left(F_{i}-\sum_{j=2}^{n} F_{i} F_{j}^{*}\right)$. In this way $F_{1}$ is eliminated from an expression for it.

For example, if we have the partition $\left\{F_{1}, F_{2}, F_{3}\right\}$, then $F_{1}=F_{1} F_{1}^{*}+F_{1} F_{2}{ }^{*}+F_{1} F_{3}^{*}=F_{1} F_{1}^{*}+\left(F_{2} F_{1}^{*}\right)^{+}+\left(F_{3} F_{1}^{*}\right)^{+}$ $=I+\left(F_{2}-F_{2} F_{2}^{*}-F_{2} F_{3}^{*}\right)^{+}+\left(F_{3}-F_{3} F_{2}^{*}-F_{3} F_{3}^{*}\right)^{+}$. 
If $F_{2}$ is the set of squares, and $F_{3}$ the set of primes, then the nth number which is neither prime nor square is equal to the $n$th element of $F_{1}=n+\left(m^{2}-m-\pi\left(m^{2}\right)\right)^{+}(n)$ $+\left(\mathrm{p}_{\mathrm{m}}-\mathrm{m}-\left[\sqrt{\mathrm{p}_{\mathrm{m}}}\right]\right)^{+}(\mathrm{n}) . \quad\left(\mathrm{n}^{2}-\mathrm{n}-\pi\left(\mathrm{n}^{2}\right)\right)^{+}(1)=2$, since $2^{2}-2-\pi\left(2^{2}\right)<1<3^{2}-3-\pi\left(3^{2}\right)$, and we find that the first such number $=1+2+3=6$.

4. We select for special attention the partition $\left\{F_{1} \mid i \in S_{n}\right\}$ expressed in the form $\left\{F_{i} \sum_{i=1}^{n} F_{i}^{*} \mid i \in S_{n}\right\}$. From the $n$ subsets of any partition $\left\{F_{i} \mid i \in S_{n}\right\}$ the monotonic increasing functions $F_{i} F_{j}^{*}$ for all $(i, j)$ in $S_{n} \times S_{n}$ may be calculated. We have shown above that $F_{i} F_{j}^{*}=\left(F_{j} F_{i}^{*}\right)^{+}$and that $F_{i} F_{j}^{*} F_{j} F_{k} \leq F_{i} F_{k}{ }^{*} \leq F_{i} F_{j}^{*} S F_{j} F_{k}^{*}$. If, conversely, the extensions of the $n^{2}$ functions $\left(F_{i} F_{j}{ }^{*}\right)$ are given, then the redundancy of information in the case just dealt with is now reflected in a demand for the consistency of the specified functions; $\mathrm{F}_{i} \mathrm{~F}_{j}{ }^{*}$ must coincide with $\left(\mathrm{F}_{\mathrm{j}} \mathrm{F}_{\mathrm{i}}{ }^{*}\right)^{+}$and $F_{i} F_{j}{ }^{*} F_{j} F_{k}{ }^{*} \leq F_{i} F_{k} \leq F_{i} F_{j}{ }^{*} S F_{j} F_{k}{ }^{*}$, for all $i, j$ and $k$ in $S_{n}$. Thus, if we are given $F_{1} F_{2}{ }^{*}=n^{2}$, and $F_{2} F_{1}^{*}=p_{n}$, there is no partition $\left\{F_{i} \mid i \in S_{3}\right\}$ which is a solution of the given equations. On the other hand, if $F_{1} F_{2}{ }^{*}=n^{2}$, and $F_{3} F_{1}{ }^{*}=p_{n}$, it seems plausible that there is a partition $\left\{F_{1}, F_{2}, F_{3}\right\}$, which is a solution of these equations. In fact, such a partition is given by $F_{1}=\left\{n+n^{2}+\pi(n-1) \mid n \in N\right\}$

$$
\begin{aligned}
& F_{2}=\{[\sqrt{n-1}]+n+\pi([\sqrt{n-1}]) \mid n \in N\} \\
& F_{3}=\left\{p_{n}+\left(p_{n}\right)^{2}+n \mid n \in N\right\} .
\end{aligned}
$$

We make the following definitions: Any subset $S$ of $S_{n} \times S_{n}$ will be called independent, if for any function $\phi$ from the elements of $S$ to non-decreasing sequences, a partition 
$\left\{F_{i} \mid i \in S_{n}\right\}$ may be found such that $F_{i} F_{j}^{*}=\phi(i, j)$ for all $(i, j)$ in $S$. Any maximal independent subset (of $S_{n} \times S_{n}$ ) will be called a partition basis (of $S_{n} \times S_{n}$ ).

The transitive closure of $R$ (the union of all positive powers of $R$ ) is denoted by $C(R)$, where $R$ is any relation (set of ordered pairs). If the transitive closure of any proper subset of $R \cup R^{-}$(the union of $R$ and its converse) is a proper subset of $C\left(R \cup R^{-}\right)$and $R$ contains no reflexive pairs, then we shall say that $R$ is circuit-free. Any maximal circuit-free subset ( of $S_{n} \times S_{n}$ ) will be called a tree (of $S_{n} \times S_{n}$ ). Note that a tree is an irreflexive, asymmetric relation whose field is $S_{n}$, and might be called an oriented tree without loops on $n$ nodes.

If $S$ is independent, then for each $\phi$ on $S$, we define $\phi$ on $S^{-}$by $\phi(j, i)=(\phi(i, j))^{+}$for all $(i, j)$ in $S$. (It is easily seen that $S$ and $S^{-}$have no common elements.) Since $F_{j} F_{i}^{*}=\left(F_{i} F_{j}^{*}\right)^{+}$for the $F_{i}$ of any partition, it follows that if $\mathrm{S}=\mathrm{V} \cup \mathrm{W}$ is independent, so is $\mathrm{V} \sim \mathcal{W}$.

If $S$ is independent and contains $(i, j)$, then $i \neq j, S$ does not contain $(j, i)$, and if $S$ contains $(j, k)$ or $(k, j)$ then it does not contain $(i, k)$ or $(k, i)$. We know that this is so because we have obtained results about the subsets of any partition which imply that $\phi$ cannot be defined arbitrarily on $S$ (e.g., $F_{i} F_{i}{ }^{*}=I$. If an independent set $S$ contains the pair $(i, i)$, then we may deduce $\phi(i, i)=I)$. If there is any subset $\left\{(i, j),\left(i_{1} i_{1}\right),\left(i_{1}, i_{2}\right), \ldots\left(i_{r}, j\right)\right\} \quad$ (all $i^{\prime} s$ and $j$ distinct) of $S \cup S^{\wedge}$, then $S$ is not independent, since if it were, $\phi$ might be chosen so that $\phi(i, j)<\phi\left(i_{1} i_{1}\right) \phi\left(i_{1}, i_{2}\right) \ldots \phi\left(i_{r}, j\right)$. Then, for the sets $F_{i}$ of some partition, $F_{i} F_{j}{ }^{*}<F_{i} F_{i 1}{ }^{*} F_{i 1} F_{i 2}{ }^{*}$ ... $F_{i r} F_{j}^{*}$, which is impossible. (Recall that $F_{i j}{ }^{*} F_{i j} \leq I$.) The preceding remarks are summarized in the statement that a necessary condition for the independence of a set is that it be circuit-free. The sufficiency of the condition may be shown by embedding $S$ in a maximal tree, applying the second part of Theorem 1, and observing that any subset of a partition basis, 
or of any independent set, is independent. It follows that every maximal independent subset of $S_{n} \times S_{n}$ is a maximal circuitfree subset of $S_{n} \times S_{n}$, so that we have proved the first part or

THEOREM 1. Every partition basis is a tree. Every tree is a partition basis.

Proof. It remains to prove the second statement. Suppose that $T$ is a tree of $S_{n} \times S_{n}$. By commuting a finite number of ordered pairs of $\mathrm{T}$, we may obtain a tree $\mathrm{T}_{1}$, such that 1 does not occur as the second member of an ordered pair of $T_{1}$, and such that $T_{1}$ contains $(j, i)$ and $(k, i)$ only if $j=k$. Expressed in the language of graph theory, $T_{1}$ is a tree all of whose branches are directed away from the root 1. If $T_{1}$ contains $(i, j)$ we shall say that $j$ is a successor of $i$, and if $C\left(T_{1}\right)$ contains $(i, j), j$ will be called a descendant of its ancestor i. It will be sufficient to show that $T_{1}$ is a partition basis. Since $T_{1}$ is a maximal circuit-free set, it is not a proper subset of an independent set, so that it will be sufficient to show that $T_{1}$ is independent.

We wish to find a partition $\left\{F_{i} \mid i \in S_{n}\right\}$, given $\phi$ and $T_{1}$, such that $F_{i} F_{j}^{*}=\phi(i, j)$ for all $(i, j)$ in $T_{1}$. It is further desirable that $\left\{F_{i} \mid i \in S_{n}\right\}$ be such that if $(i, k)$ is not in $T_{1}$, then $F_{i} F_{k}^{*}$ may be found by a general rule rather than an extensive description. Two especially convenient types of rule are:

$$
F_{i} F_{k}^{*}=F_{i} F_{j}^{*} F_{j} F_{k}^{*}=\phi(i, j) \phi(j, k)
$$

and

$$
F_{i} F_{k}^{*}=F_{i} F_{j}^{*} S F_{j} F_{k}^{*}=\phi(i, j) S \phi(j, k) \text {. }
$$

Note that if either type of rule appears, so will the other, since 
$F_{i} F_{k}^{*}=F_{i} F_{j}^{*} F_{j} F_{k}^{*}$ is equivalent to $F_{k} F_{i}^{*}=F_{k} F_{j}^{*} S F_{j} F_{i}^{*}$

$\left(F_{i} F_{k}{ }^{*}=F_{i} F_{j}^{*} F_{j} F_{k}{ }^{*}\right.$ implies $F_{k} F_{i}^{*}=P\left(F_{i} F_{k}{ }^{*}\right)^{*}=P\left(F_{i} F_{j}^{*} F_{j} F_{k}\right)^{*}$

$=P F_{k}{ }^{* * *} F_{j}{ }^{*} F_{j}^{* *} F_{i}^{* *}=P S F_{k} P F_{j}^{*} S F_{j} P F_{i}^{*}=F_{k} F_{j}^{*} S F_{j} F_{i}^{*}$, and

$F_{k} F_{i}^{*}=F_{k} F_{j}^{*} S F_{j} F_{i}^{*}$ implies $F_{i} F_{k}^{*}=\left(F_{k} F_{i}^{*}\right)^{+}=\left(\left(F_{i} F_{j}^{*} F_{j} F_{k}^{*}\right)^{+}\right)^{+}$

$\left.=F_{i} F_{j}^{*} F_{j} F_{k}^{*}\right)$. If we have such a rule then we can express

each $F_{i}$ as $\sum_{j=1}^{n} F_{i} F_{j}^{*}$, i. e. in terms of the functions in the

range of $\phi$ (which we will call basis functions).

Before obtaining such a partition, we will find a serial order of $S_{n}$ which contains $T_{1}$. Let the successors of every

$i$ with more than one successor be serially ordered, and let

$\mathrm{K}$ be the union of the chosen serial orders. Then

$\mathrm{L}=\mathrm{C}\left(\mathrm{T}_{1}\right) \cup \mathrm{K} \cup \mathrm{KC}\left(\mathrm{T}_{1}\right) \cup \mathrm{C}\left(\mathrm{T}_{1}{ }^{\wedge}\right) \mathrm{K} \cup \mathrm{C}\left(\mathrm{T}_{1}{ }^{\wedge}\right) \mathrm{KC}\left(\mathrm{T}_{1}\right)$ is a serial

order of $\mathrm{S}_{\mathrm{n}}$ containing $\mathrm{C}\left(\mathrm{T}_{1}\right)$.

This last may be put in a more comprehensible, although more prolix form by using more phrases, and focussing on a familiar serial order. Let $K$ and $L$ be represented by "Iess than". Then $i<j$ (in L) if $i$ is an ancestor of $j$, or $i<j$ (in $L$ ) if the successor of the greatest common ancestor of $i$ and $j$ which is an ancestor of $i$ is less than (in K) the successor which is an ancestor of $j$.

We will now find a partition $\left\{F_{i} \mid i \in S_{n}\right\}$ which has the desired properties. Form an infinite sequence of '1's, and for each $j$ which is a successor of 1 , interpolate $j$ 's so that if $K$ contains $(k, j)$ then $j$ precedes $k$ in the sequence, and so that the value of the function $\phi(1, j)$ at $m$ is equal to the number of $j^{\prime} s$ which precede the mth $1(m \geq 1)$. This is accomplished by interpolating a number of $j^{\prime} s$ equal to $\phi(1, j)(m)-\phi(1, j)(m-1)$ between the $m$ th and $(m-1)$ th 1 .

The key steps of an easy induction which justifies that construction are $\phi(1, j)(1)=\phi(1, j)(1)-\phi(1, j)(0)$ and $\phi(1, j)(m)$ $=\phi(1, j)(m-1)+(\phi(1, j)(m)-\phi(1, j)(m-1))$. The successors of the mth term in the sequence thus obtained are similarly interpolated between the mth and $(m-1)$ th term, and so on. 
Since the field of $T$ is finite, the process terminates with some sequence $s$. The partition $\left\{F_{i} \mid i \in S_{n}\right\}$ is determined by taking $m$ to be in the set $F_{i}$ if $i$ is the mth term of s. Then $F_{i} F_{j}^{* k}(n)=$ the number of $j^{\prime} s$ before the $n$th $i=\phi(i, j)(n)$ for all $n$ and for all $(i, j)$ in $T_{1}$. This completes the proof of the Theorem.

5. Having given a constructive proof of the second part of Theorem 1, using the auxiliary sequence $s$, we will shortly be in a position to justify the following statement of what is probably our main result:

Let there be given any oriented tree and let its nodes be labelled $1,2, \ldots, n$. To the branch $(i, j)$ let there be assigned an arbitrary function $\phi(i, j)$ (non-decreasing from $N$ to $N \cup\{0\})$. If $(i, j)$ is not a branch of the tree, define $\phi(i, j)$ by the following rules:

$$
\begin{aligned}
& \phi(i, i)=\text { the identity function I } \\
& \phi(i, j)=(\phi(j, i))^{+} \text {if }(j, i) \text { is a branch } \\
& \phi(i, k)=\phi\left(i, j_{1}\right)(S) \phi\left(j_{1}, j_{2}\right)(S) \ldots \text { (S) } \phi(j, k)
\end{aligned}
$$

if $\left(i, j_{1}\right),\left(j_{1}, j_{2}\right) \ldots\left(j_{n}, k\right)$ are branches or inverse branches of the tree. The successor function $S$ is put in parentheses to indicate that it sometimes appears and sometimes does not, as explained below. If we set $F_{i}=\sum_{j=1}^{n} \phi(i, j)$, then the ranges of $F_{1}, F_{2}, \ldots, F_{n}$ form a partition of the natural numbers, and $F_{i} F_{j}^{*}=\phi(i, j)$.

We now continue from the end of $\S 4$ to show how $F_{i} F_{k}{ }^{*}$ is found when $(i, k)$ is not in $\mathrm{T}_{1} \cup \mathrm{T}_{1} \hat{\wedge}$, and $i \neq k$. By the method of formation of the sequence, if $(i, j)$ and $(j, k)$ are in $T_{1}$, then any $k$ will precede $i$ in $s$ only if it preceded a $j$ between $k$ and $i$; therefore $F_{i} F_{k}{ }^{*}=\phi(i, j) \phi(j, k)$, and 
$F_{k} F_{i}^{*}=\phi(k, j) S \phi(j, i)$. In general, if $T_{1}$ has the subset $\left\{\left(i_{1}, i_{2}\right),\left(i_{2}, i_{3}\right), \ldots,\left(i_{m-1}, i_{m}\right)\right\}$ then

(1) $\quad F_{i 1} F_{i m}^{*}=\phi\left(i_{1}, i_{2}\right) \phi\left(i_{2}, i_{3}\right) \ldots \phi\left(i_{m-1}, i_{m}\right)$,

since every $i_{m}$ precedes an $i_{m-1}, \ldots$ preceding an $i_{3}$, preceding an $i_{2}$, preceding an $i_{1}$ in $s$. To exhibit the structure of the formula, subscripts $1,2,3, \ldots$ have been used, but formulas $1,2,3,4$ are in fact valid, when suitably interpreted, for $p \geq 1, m \geq 1$, and $\leq$ the number of elements in the field of $T$.

From (1) we find

$$
\begin{aligned}
F_{i m} F_{i 1}^{*} & =P\left(F_{i 1} F_{i m}{ }^{*}\right)^{*} \\
& =P\left(F_{i 1} F_{i 2}{ }^{*} F_{i 2} F_{i 3}{ }^{*} \cdots F_{i(m-1)} F_{i m}^{*}\right) \\
& =P F_{i m}{ }^{* *} F_{i(m-1)}{ }^{*} \ldots F_{i 3}{ }^{* *} F_{i 2}{ }^{*} F_{i 1}^{* *} F_{i 1}{ }^{*} \\
& =P S F_{i m} P F_{i(m-1)}{ }^{*} \ldots S F_{i 3} P F_{i 2}{ }^{*} S F_{i 2} P F_{i 1}^{*} \\
& =F_{i m} F_{i(m-1)}{ }^{*} \ldots F_{i 3} F_{i 2}{ }^{*} F_{i 2} F_{i 1}{ }^{*}
\end{aligned}
$$

(2) $\quad F_{i m} F_{i 1}^{*}=\phi\left(i_{m}, i_{m-1}\right) \ldots S \phi\left(i_{3}, i_{2}\right) S \phi\left(i_{2}, i_{1}\right)$.

If $(k, j)$ is in $K$ and $(i, k),(i, j)$ are in $T_{1}$, then $F_{j} F_{k}{ }^{*}=\phi(j, i) \phi(i, k)$, since no $k$ precedes a $j$ unless it also precedes an $i$ previous to that which $j$ precedes. This is likewise true if $(i, k),(i, j)$ are in $C\left(T_{1}\right)$ and $(k, j)$ is in $L$.

Suppose $T_{1}$ contains $\left(i, k_{1}\right),\left(k_{1}, k_{2}\right), \ldots,\left(k_{m-1}, k_{m}\right)$ and $\left(i, j_{1}\right),\left(j_{1}, j_{2}\right), \ldots,\left(j_{p-1}, j_{p}\right)$, and $(k, j)$ is in $L$. Then we have 
$F_{j p} F_{k m}{ }^{*}=F_{j p} F_{i}^{*} F_{i} F_{k m}{ }^{*}=\phi\left(j_{p}, j_{p-1}\right) \ldots S \phi\left(j_{2}, j_{1}\right) S \phi\left(j_{1}, i\right)$

$$
\phi\left(i, k_{1}\right) \phi\left(k_{1}, k_{2}\right) \ldots \phi\left(k_{m-1}, k_{m}\right) \text {. }
$$

Now,

$F_{k m} F_{j p}^{*}=F_{k m} F_{i}^{*}{ }^{*} F_{i} F_{j p}{ }^{*}=\phi\left(k_{m}, k_{m-1}\right) \ldots S \phi\left(k_{2}, k_{1}\right) \phi\left(k_{1}, i\right) S$

$$
\phi\left(i, j_{1}\right) \phi\left(j_{1}, j_{2}\right) \ldots \phi\left(j_{p-1}, j_{p}\right) .
$$

Thus, any $\mathrm{F}_{\mathrm{i}} \mathrm{F}_{\mathrm{k}}{ }^{*}$ may be expressed in terms of basis functions, (1) or (2) being used if $(i, k)$ or $(k, i)$ is in $C\left(T_{1}\right)$ respectively, and (4) or (3) if (i,k) or $(k, i)$ is in $\mathrm{K} \cup \mathrm{KC}\left(\mathrm{T}_{1}\right) \cup \mathrm{C}\left(\mathrm{T}_{1}{ }^{-}\right) \mathrm{K} \cup \mathrm{C}\left(\mathrm{T}_{1}{ }^{-}\right) \mathrm{KC}\left(\mathrm{T}_{1}\right)$ respectively.

Example 1 illustrates the derivation of a partition from a partition basis. An arbitrary selection of basis functions was made, except insofar as the randomness was calculated, and that, of all possible relations $T$, economy of space imposed the choice of a short one.

The basis functions were chosen to be well-known functions; the inverses of the se functions are likewise well-known. Such is not the case for functions easily enough compounded from them; an instance of this has been encountered in $\S 2$.

This is a significant psychological point, since a forced reference, when exhibiting a partition, to "the inverse of a function" rather than to a function which happens to be an inverse, is a first step in exposing the ultimately tautologous nature of the result.

Example 1. The partition basis in $\mathrm{S}_{6} \times \mathrm{S}_{6}$ is $\{(1,4),(4,3),(2,1),(2,5),(2,6)\}$. The basis functions are given by $\phi(1,4)=n^{2}, \phi(4,3)=[(n-1) / 2] \phi(2,1)=\pi(n-1)$, $\phi(2,5)=2^{n}, \phi(2,6)=n+2$ (see fig. 5 ). 


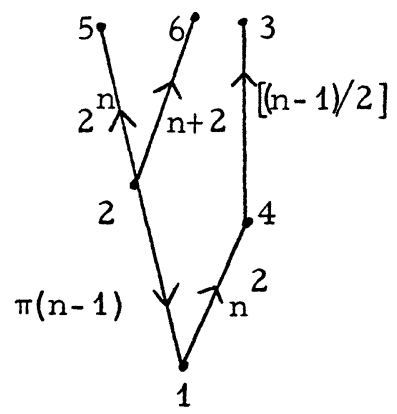

Fig. 5

Now, $\phi(4,1)=[\sqrt{n-1}], \quad \phi(3,4)=2 n, \quad \phi(1,2)=p_{n}$, $\phi(5,2)=\log _{2}(\mathrm{n}-1), \quad \phi(6,2)=\mathrm{n}-3$.

$T_{1}=\{(1,2),(2,5),(2,6),(1,4),(4,3)\} ; K=\{(4,2),(5,6)\}$,

$\mathrm{L}=$ the set of ordered pairs that corresponds to the serial order $1<4<3<2<5<6$.

The process of forming a sequence which will give us $\left\{F_{i} \mid i \in S_{6}\right\}$ is shown in Fig. 6 .

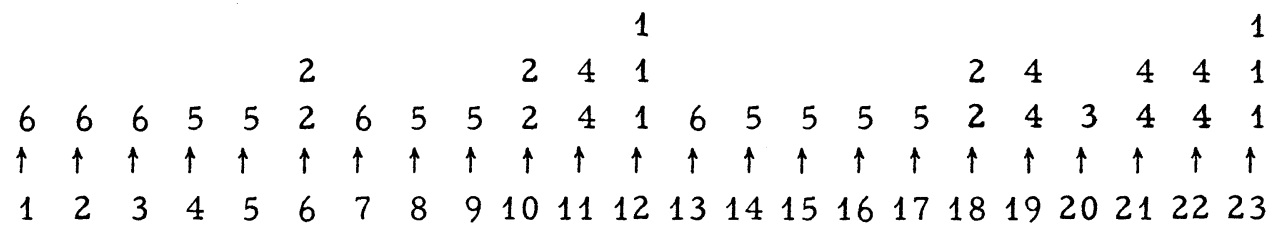

Fig. 6

The irregular spacing of the upper sequences anticipating the subsequent interpolations is, of course, an irrelevant metric property of the visual aid. Some sample calculations are $\phi(1,4)=1^{2}=1$ at 1 so that one 4 precedes the first 1 . $\phi(1,4)=2^{2}=4$ at 2 so that four $4^{\prime}$ s precede the second 1 . From the sequence 


$$
s=\{(1,6),(2,6),(3,6),(4,5),(5,5), \ldots\}
$$

we find that $F_{6}=\{1,2,3,7,13, \ldots\}$.

An example of the way in which $\mathrm{F}_{i} \mathrm{~F}_{k}{ }^{*}$ is found in terms of the basis functions follows for $\mathrm{F}_{3} \mathrm{~F}_{5}{ }^{*}$.

$$
\begin{aligned}
& F_{3} F_{4}=2 \mathrm{n}, \\
& F_{3} F_{1}{ }^{*}=\phi(3,4) S \phi(4,1)=[\sqrt{(2 n+1)-1}]=[\sqrt{2 n}], \\
& F_{3} F_{2}^{*}=\phi(3,4) S \phi(4,1) S \phi(1,2)=p([\sqrt{2 n}]+1), \\
& F_{3} F_{5}^{*}=\phi(3,4) S \phi(4,1) \operatorname{S} \phi(1,2) \phi(2,5)=2^{p([\sqrt{2 n}]+1)} .
\end{aligned}
$$

(For the printer's convenience we have written $p_{n}$ as $p(n)$.)

The partition finally obtained is $\left\{F_{i} \mid i \in S_{6}\right\}$, where

$$
F_{1}=\left\{n+p_{n}+\left[\left(n^{2}-1\right) / 2\right]+n^{2}+2^{p(n)}+\left(p_{n}+2\right) \mid n \in N\right\} \text {, }
$$$$
F_{2}=\left\{\pi(n-1)+n+\left[\left((\pi(n-1))^{2}-1\right) / 2\right]+(\pi(n-1))^{2}+2^{n}+(n+2) \mid n \in N\right\} \text {, }
$$$$
F_{3}=\left\{[\sqrt{2 n}]+p([\sqrt{2 n}]+1)+n+2 n+2^{p([\sqrt{2 n}]+1)}+(p([\sqrt{2 n}]+1)+2) \mid n \in N\right\}
$$$$
F_{4}=\left\{[\sqrt{n-1}]+p([\sqrt{n-1}]+1)+[(n-1) / 2]+n+2^{p([\sqrt{n-1}]+1)}\right.
$$$$
+(p([\sqrt{n-1}]+1)+2) \mid n \in N\},
$$$$
F_{5}=\left\{\pi\left(\left[\log _{2}(n-1)\right]\right)+\left[\log _{2}(n-1)\right]+\left[\left(\left(\pi\left(\left[\log _{2}(n-1)\right]\right)\right)^{2}-1\right) / 2\right]\right.
$$$$
\left.+\left(\pi\left(\left[\log _{2}(n-1)\right]\right)\right)^{2}+n+\left(\left[\log _{2}(n-1)\right]+3\right) \mid n \in N\right\},
$$

$F_{6}=\left\{\pi(n-3)+(n-3)+\left[\left((\pi(n-3))^{2}-1\right) / 2\right]+(\pi(n-3))^{2}+2^{n-3}+n \mid n \in N\right\}$.

If the signs indicating row summation etc. are ignored, the above is a square matrix whose $(i, j)$ th entry if $F_{i} F_{j}{ }^{*}$. It should be remembered that the customary evaluation of non-natural points 
of functions usually defined on a field wider than $\mathrm{N}$ is waived by a previous convention. Thus, $2^{n-3}=0$ for $n \leq 3$.

We have so far considered a "typical" partition whose importance lies more in the generality which its arbitrariness suggests than in its particular structure. In the following examples, elements of the partition bases and the basis functions were chosen with regard to one another to provide more elegant partitions.

Example 2. The partition basis in $S_{3} \times S_{3}=\{(1,2),(2,3)\}$. The basis functions are given by $\phi(1,2)=p_{n}, \phi(2,3)=p_{n}$. $\phi(2,1)=\phi(3,2)=\pi(n-1)$. We obtain the partition $F_{1}, F_{2}, F_{3}$, where

$$
\begin{aligned}
& F_{1}=\left\{n+p_{n}+p_{p_{n}} \mid n \in N\right\}, \\
& F_{2}=\left\{\pi(n-1)+n+p_{n} \mid n \in N\right\}, \\
& F_{3}=\{\pi(\pi(n-1))+\pi(n-1)+n \mid n \in N\} .
\end{aligned}
$$

Example 3. The partition basis in $\mathrm{S}_{\mathrm{m}} \times \mathrm{S}_{\mathrm{m}}=\{(1,2)$, $(1,3), \ldots,(1, m)\}$. The basis functions are given by $\phi(1,2)$ $=a_{2} n, \phi(1,3)=a_{3} n, \ldots \phi(1, m)=a_{m} n$, where $a_{1}=1$, and the $a_{i}$ are positive integers. After a simplification we obtain the partition $\left\{F_{i} \mid i \in S_{m}\right\}$, where, for $1 \leq j \leq m$, $F_{j}=\left\{n+\sum_{i=j+1}^{n} a_{i}+\sum_{\substack{i \neq j \\ i=1}}^{n} a_{i}\left[(n-1) / a_{j}\right] \mid n \in N\right\}$. For example, if $a_{2}=2, a_{3}=3, a_{4}=4$, then the $F_{j}$ are $\{10 n\}$, $\{n+7+8[(n-1) / 2]\},\{n+4+7[(n-1) / 3]\},\{n+6[(n-1) / 4]\}$.

Example 4. The partition basis in $\mathrm{S}_{4} \times \mathrm{S}_{4}$ is $\{(1,2),(1,3),(1,4)\}$. The basis functions are given by 
$\phi(1,2)=a_{2} n, \phi(1,3)=a_{3} n, \phi(1,4)=a_{4} n$, where the $a_{i}$ are positive irrationals, and we set $a_{1}=1$. We obtain the partition $\left\{F_{1}, F_{2}, F_{3}, F_{4}\right\}$, where

$$
\begin{aligned}
& F_{1}=\left\{n+\left[a_{2} n\right]+\left[a_{3} n\right]+\left[a_{4} n\right] \mid n \in N\right\}, \\
& F_{2}=\left\{\left[n / a_{2}\right]+n+\left[a_{3}\left(\left[n / a_{2}\right]+1\right)\right]+\left[a_{4}\left(\left[n / a_{2}\right]+1\right)\right] \mid n \in N\right\}, \\
& F_{3}=\left\{\left[n / a_{3}\right]+\left[a_{2}\left[n / a_{3}\right]\right]+n+\left[a_{4}\left(\left[n / a_{3}\right]+1\right)\right] \mid n \in N\right\}, \\
& F_{4}=\left\{\left[n / a_{4}\right]+\left[a_{2}\left[n / a_{4}\right]\right]+\left[a_{3}\left[n / a_{4}\right]\right]+n \mid n \in N\right\} .
\end{aligned}
$$

As in Example 3, we may obtain a similar partition $\left\{F_{i} \mid i \in S_{m}\right\}$. It is not hard to show that $m$ may be infinite provided that only finitely many basis functions have non-zero values at any natural point. Thus, the condition here is $\lim _{n \rightarrow \infty} a_{n}=0$.

Example 5. If the basis functions, for any partition basis in $\mathrm{S}_{\mathrm{m}} \frac{\mathrm{S}}{\mathrm{m}}$, are all of the form $\phi(i, j)(n)=n+a$, where a is a variable non-negative integer, then the subsets of the partition obtained are the congruence classes mod $m$ (in $N$ ).

I would like to thank Prof. J. Lambek for his very helpful interest and advice.

\section{REFERENCE}

1. J. Lambek and L. Moser, Inverse and Complementary Sequences of Natural Numbers, Amer. Math. Monthly, Vol. 61, No. 7, 1954, pp. 454-458.

Postscript. The article "Functions which Represent all Integers" by E.N. Gilbert has appeared in the Amer. Math. Monthly, Vol.70, No. 7, 1963, pp.736-738. The major portion of this article is a direct consequence of [1], of which the author seemed unaware. He remarks, as we do in $\S 3$, that $\left\{\sum_{i \in S_{n}} F_{j} F_{i}^{*} \mid j \in S_{n}\right\}$ is a partition of $\mathrm{N}$, and gives an interesting example of such a partition. 